\title{
1st National Congress of the Hellenic Society of Digestive Surgery
}

November 20-23, 2003, Athens, Greece

\section{Selected Abstracts}

\section{Guest Editors}

John Bramis, Athens

Gabriel Karatzas, Athens

Christos Dervenis, Athens
G. Karatzas
C. Simopoulos
C. Hatzitheophilou
D. Botsios
G. Kouraklis

Abstract Selection Committee 
Association of E2F-1 Expression and Apoptotic Index in Colorectal Carcinomas

\author{
J. Bramis, I. Papakonstantinou, P. Zacharatos, A. Kotsinas, \\ F. Sigala, P. Leonardou, V.G. Gorgoulis, E. Bastounis \\ 1st Department of Surgery, 'Laiko' Hospital, Molecular \\ Carcinogenesis Group, Department of Histology- \\ Embryology, Department of Radiology, 'Laiko' Hospital, \\ Medical School, University of Athens, Athens, Greece
}

Aim: E2F-1 is a nodal transcription factor for the G1 to $\mathrm{S}$ transition of the cell cycle that also modulates DNA synthesis and repair, mitosis and apoptosis. In colon cancer cell lines increased E2F-1 activity is functionally linked with apoptosis. In the present report we examined E2F-1 expression status and its association with the presence of apoptotic cells in colon adenocarcinomas.

Materials and Methods: Formalin fixed paraffin-embedded tissues of colorectal adenocarcinomas from 46 patients (18 females and 27 males, mean age 69 years and follow-up to 2.5 years) were employed in the analysis. Tumors were classified according to Duke's system as follows: 4 stage A, 26 stage B, 12 stage $\mathrm{C}$ and 3 stage D. Expression of E2F-1 was assessed by immunohistochemistry (antibody KH 95), following the indirect streptavidin-biotin-hyperoxide labeling method, while apoptotic cells were labeled following the TUNEL assay.

Results: In cancer areas E2F-1 expression was detected in 5\% to $80 \%$ of the cells with a mean value of $25 \%$ stained cells. The immunohistochemical signal was observed in the nuclei of the tumor cells as well as in a few stromal ones. No statistical significant associations were found between E2F-1 expression and clinicopathological data of the patients (sex, age, Duke's stage and survival). Apoptotic nuclei ranged between $0.5 \%$ up to $3.5 \%$ in tumor areas, with a mean value of $1.42 \%$. An interesting finding represented co-localization of the E2F-1 staining signal with the presence of apoptotic nuclei in the neoplastic regions, as judged from examination of serial sections. This histological finding was further strengthened by the significant statistical association between E2F-1 expression and apoptotic index (AI: \% of apoptotic nuclei) $(r=0.427, p=0.013$, Pearson test $)$ observed in our cohort of patients.

Conclusions: Expression of E2F-1 in colorectal carcinomas is not associated with clinicopathological data of the patients. Nevertheless, at the histological level there is a strong correlation between its expression and presence of apoptotic cells. This finding implies that factors modulating E2F-1 expression should be examined in the view of potential molecular therapeutic targets in colon cancer.

\section{2 \\ Virtual Colonoscopy as a Complementary Method in the Follow-Up Strategy of the Postcolectomy Patients \\ P. Leonardou ${ }^{1}$, K. Striggaris ${ }^{1}$, N. Tsavaris ${ }^{2}$, \\ I. Papakonstantinou ${ }^{3}$, A. Nikolaou $^{3}$, E. Bastounis $^{3}$, J. Bramis ${ }^{3}$ \\ ${ }^{1}$ Radiology Department, General Hospital of Athens \\ 'G. Gennimatas', ${ }^{2}$ Internal Medicine Department, \\ ${ }^{3}$ First Surgery Department, University of Athens, \\ Athens, Greece}

Purpose: With the present study we intend to evaluate virtual colonoscopy as a new diagnostic tool for the early detection of recurrences (screening) and distant metastases (staging) after complete resections for colorectal cancer.

Materials and Methods: Virtual colonoscopy was performed in 53 patients during a time of 12 to 48 months postoperative. 44 patients had partial colectomy ( $31 \mathrm{men} / 13$ women, mean age 43 years) and 9 had abdominoperineal resection and bared permanent colostomy ( $7 \mathrm{men} / 2$ women, mean age 48 years). A spiral CT examination of the colon was done after proper cleansing of the colon and its full distension with air via rectal tube. We used spiral CT (Philips Spiral CT, Tomoscan SR 7000) with the following parameters of examination: slice thickness $5 \mathrm{~mm}$, table speed $10 \mathrm{~mm}$, reconstruction index $2.5 \mathrm{~mm}$ $(120 \mathrm{KV}, 250 \mathrm{~mA})$. The patients were examined in both supine and prone position. During the supine examination, bolus injection of iodine contrast medium was introduced, to identify liver metastases. The images were transferred to a separate workstation (Philips Easy Vision) for postprocessing, 3D rendering and endoluminal view. The examination was undertaken as part of the routine follow-up of the patients and the results were compared to the ones of conventional colonoscopy as well as the rest laboratory findings.

Results: Recurrence was detected in 4 patients (8\%). In 6 instances, conventional colonoscopy was not diagnostic, because of the endoscope inability to pass through the entire colon, whereas

\begin{tabular}{ll}
\hline KARGER & ( ) 2004 S. Karger AG, Basel \\
0253-4886/04/0211-0013\$21.00/0 \\
$\begin{array}{l}\text { Fax +4161306 1234 } \\
\text { E-Mail karger@karger.ch } \\
\text { www.karger.com }\end{array}$ & $\begin{array}{l}\text { Accessible online at: } \\
\text { www.karger.com/dsu }\end{array}$
\end{tabular}


2 patients with colostomy refused the examination. In all these 8 cases (15\%), virtual colonoscopy was successfully completed. False negative result was encountered in one case, after a low anterior resection, where the mass developed very low in the rectum and was revealed by conventional colonoscopy. No liver metastasis was detected.

Conclusions: The examination is well tolerated by the patients, especially the ones with permanent colostomy. Furthermore, the injection of contrast medium during the examination is important for staging and especially for the detection of liver metastases. Since the follow-up of patients after curative resections for colorectal cancer remains controversial, virtual endoscopy seems to be a reliable, safe and cost-effective imaging technique providing an excellent alternative to conventional colonoscopy.

\section{3 \\ Molecular Indexes in the Patients with Large Intestinal Cancer}

D.B. Mylonaki, E. Hatziyianni, N. Memos, O. Kalathaki, H. Markoyiannakis, G. Androulakis

The use of molecular indexes as prognostical tools had, in the past ambigual results. The theory that specific genes which deal with apoptosis when they are expressed or co-expressed with success predict the recurrence and overall survival and this is new and interesting research. We have studied immuno-histochemical pathological tissues from 35 patients who had suffered from large intestinal cancer. We correlated the expression and the co-expression of indexes p53, bcl-2, bax and HLA-DR with several clinicopathological parameters and overall survival.

From our research we found that there is over expression of genes p53, bax, bcl-2 and HLA-DR, the pathological tissue of large intestine. The synchronized expression of genes $\mathrm{p} 53$, bcl-2 and bax did not appear to be useful prognostical factor. Also the simple over expression of gene p53 did not appear to affect statistically importantly the prognosis over the patients in the stage III.

In the other side it was found that the antigen HLA-DR was over expressed in $88.9 \%$ of the biopsies of the large intestinal cancer.

The use of several indexes and especially of the apoptosis genes in the pathological tissue of large intestine, expresses reliably the apoptotical role of the cancer cells of the tumor in patients with large intestinal cancer, but it was not proved that it influences the overall expression and over expression of the previous mentioned indexes in the skin.
4

\section{Early Enteral Nutrition in Digestive Surgery-Induced Oxidative Stress}

\author{
K. Kotzampassi ${ }^{1}$, G. Kolios ${ }^{2}$, S. Heliadis ${ }^{3}$, \\ G. Metaxas ${ }^{1}$, D. Paramythiotis ${ }^{1}$, E. Eleftheriadis ${ }^{1}$ \\ Departments of ${ }^{1}$ Surgery and ${ }^{3}$ Biological Chemistry, \\ University of Thessaloniki, Thessaloniki, ${ }^{2}$ Department of \\ Gastroenterology, University of Crete, Heraklion, Greece
}

Anesthesia and surgical trauma are considered as oxidative stress effectors, by reducing the intrinsing ability of the organism to fight against free radicals. In particular, manipulation of visceral organs during abdominal operations leads to intestinal dysmotility, which is considered to be mediated by the tissue inflammatory reaction and the oxidative stress by-products and, through the NO pathway leads to increased intestinal permeability and bacterial translocation.

The present study was designed to quantify the oxidative stress after a standard abdominal operation and to evaluate the influence of enteral nutrition on it.

A total of 60 male Wistar rats were used. Twenty of them served as controls, while the remaining 40 were subjected to an extended midline laparotomy, after which a tube-gastrostomy was performed, the distal end of the catheter exteriorized through a subcutaneous tunnel to the rats' neck (major trauma). Two hours later, operated rats were allocated to receive, through gastrostomy tube, either enteral feeding (Fresubin-HP Energy, Fresenius-Kabi, $2 \mathrm{ml} / \mathrm{h}, 75 \mathrm{kcal} / \mathrm{d}$ ) or water, for $24 \mathrm{~h}$. Following this, all rats were subjected to blood sampling for NO, SOD and GPx and to liver tissue sampling for MDA and energy charge (EC) determination.

Operation itself was found to increase NO and MDA (oxidative stress markers) and to decrease SOD, GPx (self-defense antioxidative system markers), as well as liver tissue EC, in relation to controls $(p=0.0001)$. Similarly, rats received enteral nutrition exhibited an increase of NO and MDA and a decrease of SOD, GPx and EC, but to a significantly lesser degree $(p=0.0001)$, in relation to placebo feeding rats.

\begin{tabular}{|c|c|c|c|c|c|}
\hline & NO & MDA & SOD & GPx & $\mathrm{EC}$ \\
\hline Controls & $34.70 \pm 3.70$ & $0.77 \pm 0.14$ & $5.52 \pm 0.34$ & $6,314 \pm 377$ & $739 \pm 30$ \\
\hline $\begin{array}{l}\text { Operation }+ \\
\text { water }\end{array}$ & $89.61 \pm 15.03$ & $1.39 \pm 0.08$ & $2.15 \pm 0.33$ & $2,460 \pm 160$ & $487 \pm 45$ \\
\hline $\begin{array}{l}\text { Operation }+ \\
\text { enteral } \\
\text { feeding }\end{array}$ & $49.56 \pm 8.78$ & $1.29 \pm 0.10$ & $2.51 \pm 0.30$ & $2,971 \pm 339$ & $579 \pm 53$ \\
\hline
\end{tabular}

It is thus concluded that early enteral feeding significantly ameliorates oxidative stress induced by anesthesia and surgery and exhibits a positive influence on hepatic energy charge. 


\section{5}

\section{Phospholipids for Post-Operative and/or Peritonitis-Induced Adhesions Prevention}

\author{
K. Kotzampassi, D. Paramythiotis, G. Metaxas, \\ K. Kapoutzis, H. Doumaki, E. Eleftheriadis \\ Department of Surgery, University of Thessaloniki, \\ Thessaloniki, Greece
}

Phospholipids (PL) are natural constituents of abdominal cavity fluid and cell membranes. They are synthesized and secreted by mesothelial cells and exhibit similar functions to the lung surfactant: they provide excellent lubrication and anti-adhesion properties, thus they have been used experimentally for adhesions prevention, but the results are a little confused.

The present study was designed to investigate whether intraperitoneal application of PL is capable of reducing post-operative adhesions in two standardized experimental models of abdominal trauma and peritonitis.

For the first model a total of 20 Wistar rats were used, which were subjected to a standard peritoneal and cecum abrasion trauma (nonseptic operation). For the second model, 40 rats were subjected to cecum ligation and triple puncture, for peritonitis induction (septic operation). Following non-septic or septic injury, a bolus of $3 \mathrm{ml}$ of PL (12 mg/ml, Vitalipid, Fresenius, Hellas, $120 \mathrm{mg} / \mathrm{kg} \mathrm{BW}$ ) or $\mathrm{NaCl}$ was given intraperitoneously, and the abdominal trauma was closed. Seven days later, the adhesions intensity was evaluated blindly, and their area (in $\mathrm{mm}^{2}$ ) was plotted by the use of a digital camera and a PC.

There were no 24-hours deaths in the first setting of experiments (non-septic operation), while in the latter there were 4 out of 20 and 7 out of $20(\mathrm{p}=0.01)$ in PL and placebo treated rats, respectively. Additionally, there was a significant difference between the two groups, in respect to the intensity and the area of adhesions $(\mathrm{p}=0.001)$.

\begin{tabular}{llllll}
\hline & \multicolumn{2}{l}{ Non-septic operation } & & \multicolumn{2}{l}{ Peritonitis } \\
\cline { 2 - 3 } \cline { 5 - 6 } & $\mathrm{NaCl}$ & $\mathrm{PL}$ & & $\mathrm{NaCl}$ & $\mathrm{PL}$ \\
\hline Adhesions (intensity) & $10.8 \pm 4.2$ & $4.8 \pm 2.5$ & & $11.2 \pm 4.5$ & $4.2 \pm 2.1$ \\
Area $\left(\mathrm{mm}^{2}\right)$ & $213 \pm 62$ & $75 \pm 37$ & & $254 \pm 78$ & $68 \pm 32$
\end{tabular}

It is thus concluded that PL prevent adhesion formation by means of their lubrication and anti-adhesive properties, as previous studies have also shown. However, the reduction of mortality rate on PL-treated septic group could be suggesting another pathway of action, possibly through immuno-modulation. Thus, the research is now diverted towards this direction.

\section{6}

\section{Scintigraphic Imaging with Tc 99m CEA-SCAN Monoclonal Antibody in the Follow-Up of Recurrence of Patients with Colorectal Cancer}

\author{
A. Zissimopoulos ${ }^{1}$, K. Tsamakidis², G. Ramandanis ${ }^{4}$, \\ N. Baziotis ${ }^{3}$ \\ ${ }^{1}$ Democritos University of Thrace, Thrace, \\ ${ }^{2}$ Gastroenterological Department, \\ ${ }^{3}$ Nuclear Medicine Department, 'B' Surgical Clinic, \\ St. Savas Anticancer Hospital, Athens, Greece
}

Aim: The aim of this study is the evaluation of the use of Tc $99 \mathrm{~m}$ arcitumomab (CEA-SCAN) for the detection of the recurrence of patients with colorectal cancer.

Patients and Methods: We studied 28 patients (16 male, 12 female) aged from 43-68 years (mean age 56.4 years) with a histological confirmed diagnosis of colorectal carcinoma. After resection 6 had known metastatic disease by CT and biopsy (group A), 18 with no evidence of disease in whom metastasis or recurrence was suspected based upon elevated serum CEA levels (group B), and 4 with positive CT suspected recurrence of the disease (group C). Every patient had an IV administration of $30 \mathrm{mCi}$ Tc 99m CEA-SCAN (Immunomedics) and a whole body planar and tomographic in gamma Camera 4 and 24 hours post injection. Our results were compared with the results of other anatomical imaging methods.

Results: From group A all the patients had positive CEA-SCAN (Hepatic and abdomen lesions).

From group B 14 patients had positive imaging with hepatic, abdomen and pelvic recurrence or/and metastases (78\%). From group $\mathrm{C} 2$ had positive scan and these results were verified by biopsy.

Conclusions: Tc $99 \mathrm{~m}$ CEA-SCAN is a useful diagnostic method in the management of patients with colorectal cancer. It helps in the follow-up of patients with recurrence or metastases especially in those with suspicion of the recurrence with elevated serum CEA levels.

\section{7 Serum Levels of Soluble Fas (CD95) in
Pancreatic Cancer Patients}

S. Anagnostoulis, H. Bolanaki, A. Efthimiadou, A. Polychronidis, A. Karayiannakis, C. Simopoulos

Second Department of Surgery and Department of Physiology, Democritos University of Thrace, Thrace, Medical School, Alexandroupolis, Greece

Background/Aim: Fas (CD95) is a membrane-bound type I protein that induces apoptosis upon Fas ligand (FasL) binding. Experimental studies have shown that several solid tumors secrete soluble Fas which might compete with tumor cell-surface Fas for T-cell/NK cell-surface FasL, thus enabling tumor cells to evade from immunosurveillance by tumor-infiltrating lymphocytes. In this study we evaluated soluble Fas (sFas) concentrations in the serum of pancreatic cancer patients and examined their clinical significance. 
Patients and Methods: Serum levels of sFas were determined by an enzyme immunoassay in 50 healthy control subjects and in 76 patients with pancreatic cancer before and one month after surgery. The association between preoperative sFas levels, clinicopathological features and patient survival, and their changes following surgery were evaluated.

Results: Preoperative sFas levels in pancreatic cancer patients were significantly higher when compared with those in healthy controls. These levels correlated significantly with the presence of lymph node and distant metastases and advanced disease stage. Patients with elevated sFas levels had a shorter survival when compared with those with normal sFas levels. Preoperative serum sFas levels decreased significantly after curative tumor resection whereas they remained unchanged after non-resectional surgery.

Conclusions: Pancreatic cancer patients have elevated serum sFas levels compared with healthy controls. These levels correlate with the presence of lymph node and distant metastases and advanced disease stage whereas elevated sFas levels are a poor prognostic factor for patient survival.

\section{8}

\section{ESPAC-1: Final Results of a European, Randomised Controlled Trial to Assess the Roles of Adjuvant Chemotherapy and Adjuvant Chemoradiation in Resectable Pancreatic Cancer}

\author{
C. Dervenis', J.P. Neoptolemos², D.D. Stocken ${ }^{3}$, H. Friess ${ }^{4}$, \\ C. Bassi ${ }^{5}$, H. Hickey ${ }^{2}$, H.G. Beger ${ }^{6}$, E. Hatzitheoklitos ${ }^{7}$, \\ M.W. Büchler ${ }^{4}$ on Behalf of the ESPAC group \\ ${ }^{1}$ Agia Olga, Athens, Greece; ${ }^{2}$ Liverpool University, \\ Liverpool, ${ }^{3}$ University of Birmingham, Birmingham, UK; \\ ${ }^{4}$ University of Heidelberg, Heidelberg, Germany; \\ ${ }^{5}$ University of Verona, Verona, Italy; ${ }^{6}$ University Hospital of \\ Surgery, Ulm, Germany; ${ }^{7}$ Papageorgiou Hospital, \\ Thessaloniki, Greece
}

Pancreatic cancer affects $8-12$ per 100,000 population per year in Europe and North America. Although resection improves prognosis, survival following surgery is poor with 5-year survival rates of $10-15 \%$.

ESPAC-1 is the largest randomised study to date designed to simultaneously investigate the roles of adjuvant chemoradiation (40 Gy + $5 \mathrm{FU})$ and adjuvant chemotherapy ( $5 \mathrm{FU} / \mathrm{FA})$ in patients with resected pancreatic cancer. Previously GITSG showed a survival benefit for chemoradiation in a 43 patient study; EORTC showed no significant advantage for chemoradiation in a 114 patient study, the Norwegian Pancreatic Group showed no overall survival benefit for chemotherapy in a 47 patient study and a Japanese group showed no significant benefit for chemotherapy in a 158 patient study.

ESPAC-1 randomised 550 patients with ductal adenocarcinoma including $111(20 \%)$ with R1 tumours. Preliminary analyses were published with 314 (58\%) deaths recently updated to 435 (79\%) deaths with median follow-up of 45 (IQR 28-64) months for the primary endpoint of 2-year survival. This final analysis has confirmed no survival benefit for chemoradiation (median survival 15.5 months with chemoradiation vs. 16.7 months without, $\mathrm{p}=0.078$ ) with overall 5-year survival rates of $10 \%$ with chemoradiation and $20 \%$ without. Evidence of a chemotherapy benefit exist (median survival 21.6 months with chemotherapy vs. 14.8 months without, $\mathrm{p}<0.001$ ) with overall 5-year survival rates of $23 \%$ with chemotherapy and $10 \%$ without. The chemotherapy effect has reduced magnitude in 289 patients randomised in the original $2 \times 2$ factorial design (median survival 20.1 months with chemotherapy vs. 15.5 months without, $\mathrm{p}=0.009$ ) with 5 -year survival rates of $21 \%$ with chemotherapy and $8 \%$ without.

\section{9 \\ Gut Permeability in Experimental Obstructive Jaundice: Cellular and Biochemical Alterations of the Intestinal Mucosa and Influence of Gut Regulatory Peptides Bombesin and Neurotensin}

\begin{abstract}
S.F. Assimakopoulos ${ }^{1,2}$, C.D. Scopa ${ }^{2}$, A. Charonis ${ }^{3}$, C. Georgiou 4 , V. Nikolopoulou ${ }^{1}$, C. Vagianos ${ }^{5}$

Departments of ${ }^{1}$ Internal Medicine, ${ }^{2}$ Pathology, ${ }^{5}$ Surgery, School of Medicine and ${ }^{4}$ Department of Biology, Division of Genetics, Cell and Developmental Biology, University of Patras, Patras, ${ }^{3}$ Laboratory of Cell Biology, Center for Basic Research, Foundation of Biomedical Research of the Academy of Athens, Athens, Greece
\end{abstract}

Aim: The present study investigates the influence of experimental obstructive jaundice and gut regulatory peptides bombesin (BBS) and neurotensin (NT) on: (1) the expression of the tight junctionassociated protein occludin in the intestinal epithelium, (2) intestinal epithelial cell apoptosis and proliferation in crypts and (3) intestinal oxidative stress.

Materials and Methods: Seventy adults male Wistar rats were randomly divided into 5 groups: controls, sham operated, bile duct ligated (BDL), BDL + BBS $(10 \mu \mathrm{g} / \mathrm{kg}$, s.c. $\times 3), \mathrm{BDL}+\mathrm{NT}$ $(300 \mu \mathrm{g} / \mathrm{kg}$, i.p.). At the end of the study period, on day 10 , total bilirubin and endotoxin concentrations in portal and aortic blood were measured. Tissue sections from the terminal ileum were examined for immunohistochemical expression of occludin in the intestinal mucosa, epithelial cell apoptosis (TUNEL assay) and proliferation (mitotic index) in intestinal crypts and intestinal mucosa morphometry. In addition, ileal mucosa was analyzed for DNA and protein content. Intestinal oxidative state was estimated by determination of lipid peroxidation, protein oxidation and thiol redox state [reduced glutathione (GSH), oxidized glutathione (GSSG), total non-protein mixed disulfides (NPSSR), protein thiols (PSH) and protein disulfides (PSSP)] on tissue homogenates from the terminal ileum.

Results: Experimental obstructive jaundice resulted in portal and aortic endotoxaemia. In the BDL group there was regional loss of occludin expression in the intestinal epithelium, which was most profound at the upper third of the villi. Intestinal cell apoptosis in crypts was significantly increased, while mitotic activity was reduced. This effect was accompanied by induction of mucosal atrophy, as evidenced by morphometrical analysis and decreased DNA and protein content. Moreover, obstructive jaundice induced intestinal oxidative stress demonstrated by increased lipid peroxidation, protein oxidation, GSSG, NPSSR, PSSP and reduction of GSH. Administration of 
BBS or NT significantly reduced portal and systemic endotoxaemia. These agents restored occludin expression in the intestinal epithelium to the control state and significantly reduced apoptosis, oxidative stress and mucosal atrophy.

Conclusions: Intestinal barrier dysfunction in experimental obstructive jaundice is associated with regional loss of occludin expression, increased apoptosis and oxidative stress in the intestinal epithelium. Gut regulatory peptides BBS and NT exerting beneficial effects on these cellular and biochemical alterations of the intestinal mucosa prevent portal and systemic endotoxaemia.

10

Intravenous N-Acetylcysteine, Ascorbic Acid and Selenium-Based Anti-Oxidant Therapy in Severe Acute Pancreatitis

\section{I.T. Virlos, A.K. Siriwardena \\ Department of Surgery, Manchester Royal Infirmary,} Manchester, UK

Background: To observe outcome in a cohort of patients with severe acute pancreatitis receiving multiple anti-oxidant therapy.

Methods: An observational study was carried out in 46 consecutive patients with acute pancreatitis fulfilling current Atlanta consensus criteria for severe disease. All patients received multiple anti-oxidant therapy based on intravenous selenium, $\mathrm{N}$-acetylcysteine and ascorbic acid plus $\beta$-carotene and $\alpha$-tocopherol delivered via nasogastric tube. Principal outcomes were the effect of anti-oxidant supplementation on anti-oxidant levels, morbidity and mortality in patients on anti-oxidant therapy, case-control analysis of observed survival compared to predicted survival derived from logistic organ dysfunction score (LODS), logistic regression analysis of factors influencing outcome and side-effect profile of anti-oxidant therapy.

Results: Paired baseline and post-supplementation data were available for 25 patients and revealed that anti-oxidant supplementation restored vitamin $\mathrm{C}(\mathrm{P}=0.003)$ and selenium $(\mathrm{P}=0.028)$ toward normal. In univariate survival analysis, patient survival to discharge was best predicted by admission APACHE-II score with relative risk of death increasing $12.6 \%$ for each unit increase (95\% CI: $6.0 \%$ to $19.6 \%$ ). The mean LODS calculated on admission to hospital was 3.7 (s.e.m. 4.1) giving a predicted mortality for the cohort of $21 \%$. The observed in-hospital mortality was $43 \%$.

Conclusion: Case-control analyses do not appear to demonstrate any benefit from the multiple anti-oxidant combination of selenium, $\mathrm{N}$-acetylcysteine and ascorbic acid in severe acute pancreatitis.
11

\section{Pyrrolidine Dithiocarbamate Modulates the Severity of Cerulein Induced Murine Acute Pancreatitis}

\author{
I.T. Virlos', A.K. Siriwardena ${ }^{1}$, S. Cuzzocrea ${ }^{2}$ \\ ${ }^{1}$ Hepatobiliary Unit, Department of Surgery, Manchester \\ Royal infirmary, Manchester, UK; ${ }^{2}$ Institute of \\ Pharmacology, University of Messina, Messina, Italy
}

Background: The nuclear factor- $\mathrm{B}(\mathrm{NF}-\kappa \mathrm{B})$ is a transcription factor, which plays a pivotal role in the induction of genes involved in the response to injury and inflammation. Dithiocarbamates are antioxidants, which are potent inhibitors of NF- $\mathrm{BB}$. This study tests the postulate that pyrrolidine dithiocarbamate (PDTC) attenuates experimental acute pancreatitis.

Methods: Expression of the adhesion molecule ICAM-1, nitrotyrosine, myeloperoxidase, malondialdehyde, amylase, lipase and histologic evidence of lung and pancreas injury were measured in three groups: control (saline only), cerulein and PDTC plus cerulein.

Results: Intraperitoneal injection of cerulein in mice resulted in severe, acute pancreatitis characterised by oedema, neutrophil infiltration, tissue haemorrhage and necrosis and elevated serum levels of amylase and lipase. Infiltration of pancreatic and lung tissue with neutrophils (measured as increase in myeloperoxidase activity) was associated with enhanced lipid peroxidation (increased tissue levels of malondialdehyde). Immunohistochemical examination demonstrated a marked increase in immunoreactivity for nitrotyrosine and poly (ADP-ribose) synthetase (PARS) in the pancreas and lung of cerulein-treated mice. In contrast, the degree of (1) pancreas and lung injury, (2) upregulation/expression of ICAM-1, (3) staining for nitrotyrosine and PARS and (4) lipid peroxidation was markedly reduced by pre-treatment with PDTC.

Conclusion: The findings demonstrate that prevention of the activation of NF- $\mathrm{\kappa B}$ by PDTC ameliorates experimental murine acute pancreatitis and provide important insight into the molecular biology of acute pancreatitis.

This work was supported by the 2001 Travel Fellowship of the Pancreatic Society of Great Britain and Ireland.

12

\section{CT Colonography (Virtual Colonoscopy): Comparison of Multislice CT with Colonoscopy in the Detection of Colonic Tumors}

V. Sotiropoulos, D. Karagiannis, K. Paraskeuopoulos,
N. Mathou, K. Konstantinidis, N. Bontozoglou

Athens Medical Center, Athens, Greece

Purpose: The recent introduction of multislice CT has given new perspectives in the use of CT colonography. The purpose of this study is the assessment of the accuracy of CT colonography in comparison to colonoscopy in the detection of colorectal cancer. 
Materials and Methods: Twenty-seven patients underwent CT colonography and colonoscopy. In all patients the evaluation with CT colonography was complete in the supine and prone position. In 8 out of 27 patients colonoscopy was complete, while in 19 incomplete. The performance of CT colonography and colonoscopy was assessed in 98 colonic segments evaluated with both techniques.

Findings: Colonoscopy detected 6 colon cancers and 15 polyps. The sensitivity of CT colonography was $100 \%$ for colon cancers, $100 \%$ for polyps $>10 \mathrm{~mm}, 100 \%$ for polyps $6-9 \mathrm{~mm}$ and $36.4 \%$ for polyps $<6 \mathrm{~mm}$. The corresponding specificities were $100 \%, 100 \%$, $96 \%$ and $95.8 \%$. The negative predictive value of CT colonography for cancers and polyps $>6 \mathrm{~mm}$ was $100 \%$, while for polyps $<6 \mathrm{~mm}$ it was $84.6 \%$.

Conclusion: CT colonography with the use of the new multislice technique presents excellent sensitivity and specificity in the detection of colorectal cancer as well as polyps $<5 \mathrm{~mm}$.

\section{3 \\ CT Evaluation of Gastrointestinal (GI) Tract Perforation}

V. Maniatis, H. Chrysikopoulos, A. Roussakis, E. Vardaki,

R. Efthimiadou, D. Savidou, J. Pappas, J. Andreou

CT Department 'Hygeia' Hospital, ' $\mathrm{CT}$ Department

'G. Gennimatas' General State Hospital, Athens, Greece

Purpose: To assess the value of CT in the diagnosis of GI tract perforation.

Materials and Methods: We retrospectively reviewed the CT scans of 146 patients with proven GI tract perforation to determine the signs of GI tract perforation. 80 men and 66 women were included (17-96 years old). CT diagnosis of GI tract perforation was based on (a) direct findings of extraluminar air or gastrografin and (b) indirect findings of an abscess or an inflammatory mass surrounding an appendicolith or a bowel wall-related phlegmon or abscess with fluid in the mesentery or surrounding radiopaque foreign body.

Results: There were 130 true-positive and 16 false-negative cases (sensitivity: 89\%). Diagnosis of GI tract perforation was based on direct and indirect findings in 108 and 22 cases respectively. Levels of perforation were the esophagus (3), stomach (9), duodenum (27), small bowel (25), appendix (8), and colon (74). Causes of perforation were peptic ulcer (26), foreign body (9), trauma (11), iatrogenic (14), appendicitis (8), diverticulitis (43), Crohn disease (9), neoplasm (16), ischemia (6), unknown (3), and coprostasis (1). Level and cause were correctly predicted in 105 and 86 cases, respectively.

Conclusions: CT is a valuable method in the diagnosis of GI tract perforation. The diagnosis can be established rapidly, without patient preparation. Level and cause of perforation can be usually predicted.
14

\section{Computer-Assisted Laparoscopic Repair of 'Upside-Down' Stomach with the da Vinci ${ }^{\mathrm{TM}}$ System}

C. Braumann, C. Menenakos, J.I. Rueckert, J.M. Mueller, C.A. Jacobi

Department of General, Visceral, Vascular and Thoracic Surgery, Medical Faculty Charité, Humboldt University, Berlin, Germany

Purpose: Although laparoscopic and endoscopic procedures have changed many aspects of surgical care in a revolutionary way, the inherent limitations of traditional laparoscopic surgery may cause certain difficulties during the performance of advanced laparoscopic operations. Telerobotic surgical systems attempt to provide a solution to these problems. In this article we present the first four cases of telerobotic assisted hiatal hernia repair with intrathoracic 'upside-down' stomach using the da Vinci ${ }^{\mathrm{TM}}$ robotic system.

Patients/Methods: Four Patients ( 3 males and 1 female) with hiatal hernia and intrathoracic stomach were treated operatively using the da Vinci ${ }^{\mathrm{TM}}$ robotic system. All parts of the operation including tissue dissection, hiatoplasty and anterior hemifundoplication were performed by the robotic system.

Results: All the operations were performed without complications. We found great advantages using the da Vinci ${ }^{\mathrm{TM}}$ system mostly in terms of better orientation of the surgeon due to the three dimensional imaging, and better performance of surgical tasks such as tissue dissection and suturing due to the wrist action of the robotic arms providing six degrees of freedom plus grasping.

Conclusions: Telerobotic assisted hiatal hernia operation is feasible with many advantages compared to the traditional laparoscopic approach especially during dissection in the mediastinum in patients with intrathoracic stomach. Technological evolution will hopefully diminish the current problems associated with robotic surgery.

15 Obesity is not a Contraindication to
Laparoscopic Nissen Fundoplication

D.P. Korkolis, E.E. Zervos, M.M. Murr, A.S. Rosemurgy

Department of Surgery, University of South Florida, Tampa, Fla., USA

Purpose: Obesity was traditionally considered to be a contraindication to anti-reflux surgery. This study was undertaken to determine the effect of preoperative Body Mass Index (BMI) on outcome after laparoscopic Nissen fundoplication.

Materials and Methods: 286 patients suffering from gastroesophageal reflux underwent laparoscopic Nissen fundoplication during the period 1998-2003. Preoperative BMI was correlated with satisfaction after fundoplication and willingness to undergo fundoplication again, if necessary. These parameters were scored by patients utilizing a Likert Scale at each clinic visit through completion of a questionnaire. Data are reported as: median (range; mean \pm S.D.). 
Results: 286 patients, 132 men and 154 women, of age 51.6 years \pm 14.4 , underwent laparoscopic Nissen fundoplication with a preoperative median BMI of 27 (range: $18-40 ; 27.1 \pm 4.2$ ) and a median DeMeester Score of 32 (range: 6-362; 52.0 \pm 26.0 ). 224 (78\%) patients reported excellent or very good results, whereas 230 (80\%) patients would undergo the fundoplication again, if necessary. Fair and poor outcomes were generally due to dysphagia. BMI did not correlate with outcome $\left(\mathrm{r}^{2}=0.0118\right.$, slope $\left.=0.0265\right)$ or willingness to undergo the fundoplication again, if necessary $\left(r^{2}=0.015\right.$, slope $=0.0116$ ).

Conclusions: Over a wide range of BMI, always less than 40, outcome after laparoscopic Nissen fundoplication was not impacted by BMI. Under a BMI of 40, obesity is not a contraindication for laparoscopic Nissen fundoplication, thereby extending the applicability of fundoplication.

\section{6 \\ Laparoscopic Heller Myotomy with Intraoperative Endoscopy}

D.P. Korkolis, E.E. Zervos, S.B. Goldin, A.S. Rosemurgy

Department of Surgery, University of South Florida,

Tampa, Fla., USA

Purpose: The optimal approach to intraoperative evaluation of the adequacy of laparoscopic Heller myotomy for the treatment of achalasia is still under debate. The aim of this study was to show that laparoscopic Heller myotomy together with intraoperative endoscopy achieves an adequate myotomy and limits the risk of postoperative gastroesophageal reflux.

Materials and Methods: 156 patients suffering from symptomatic achalasia of the esophagus underwent laparoscopic Heller myotomy in conjunction with intraoperative endoscopy during the period 1993-2003. These were 71 men and 85 women with a median age of 47 years (range 17-62 years). They were prospectively followed-up for a period of $23 \pm 2.2$ months.

Results: Preoperative symptoms included dysphagia (100\%), emesis/regurgitation (68\%), heartburn (58\%) and postprandial chest pain $(49 \%)$. Following myotomy, significant improvement $(\mathrm{p}<0.0001)$ was seen in dysphagia $(43 \%)$, postprandial chest pain $(13 \%)$ and emesis/regurgitation (9\%). Mean reflux score (scale 0 to 5 ) improved from $3.7 \pm 0.3$ to $1.5 \pm 0.2(\mathrm{p}<0.0001)$. Total improvement in symptomatology was reported in $96 \%$ of patients. Partial fundoplication was used only in 12 patients as part of hiatus reconstruction $(n=8)$ or repair of esophageal perforation $(n=4)$.

Conclusions: Intraoperative endoscopy during laparoscopic Heller myotomy guides the extent and adequacy of myotomy. By utilizing a focused dissection with preservation of the natural antireflux mechanisms around the gastroesophageal junction and by limiting the extent of myotomy along the cardia, the use of concomitant fundoplication is avoided and the risk of postoperative reflux symptoms is minimized.

\section{7}

\section{Portacaval Shunt with Small-Diameter \\ $8 \mathrm{~mm}$ Prosthetic H-Graft: Definitive \\ Treatment for Variceal Hemorrhage from Portal Hypertension}

\author{
D.P. Korkolis, E.E. Zervos, L.C. Carey, A.S. Rosemurgy \\ Department of Surgery, University of South Florida, \\ Tampa, Fla., USA
}

Purpose: Partial portal decompression has become an attractive option in the treatment of complicated portal hypertension. This study was undertaken to report long-term follow-up after partial portal decompression utilizing $8 \mathrm{~mm}$ prosthetic $\mathrm{H}$-graft portacaval shunts (HGPCS).

Materials and Methods: 110 consecutive patients suffering from portal hypertension and having a history of variceal hemorrhage and failure of conservative management, underwent HGPCS, during the period 1988-1998. Prospective follow-up recorded efficacy of partial portal decompression, shunt patency, morbidity of shunting, and overall survival.

Results: 70 males and 40 females, whose average age was $54 \pm 15$ years, underwent shunting. Cirrhosis was due to alcohol abuse in $65 \%$ of cases. $14 \%$ of patients were in Child's Class A, $55 \%$ in Child's Class B, and 31\% in Child's Class C. HGPCS was undertaken as emergency in $20 \%$, urgently in $13 \%$, and electively in $67 \%$ of patients. Shunting decreased portal pressure in all patients $(30 \pm 5.3 \mathrm{mmHg}$ to $19.9 \pm 5.5 \mathrm{mmHg} ; \mathrm{p}<0.001)$. Early and late thrombosis was reported in $6.4 \%$ and $3.6 \%$, respectively. Late rebleeding occurred in $5.4 \%$ of cases. Perioperative (30-day) mortality was $11.8 \%$ and was highest for patients with Child's Class C. Three-year survival was $55 \%$, whereas five-year survival reached $45 \%$.

Conclusions: Partial portal decompression is achieved with small-diameter $8 \mathrm{~mm} \mathrm{H}$-graft portacaval shunting. Rebleeding, shunt occlusion and encephalopathy are uncommon. In patients with severe alcoholic cirrhosis, H-graft portacaval shunting offers a definitive treatment for variceal hemorrhage from portal hypertension.

18

Transjugular Intrahepatic Portasystemic

Stent Shunts versus Small-Diameter Prosthetic H-Graft Portacaval Shunts for the Treatment of Bleeding Varices: A Prospective Study

\section{D.P. Korkolis, E.E. Zervos, L.C. Carey, A.S. Rosemurgy \\ Department of Surgery, University of South Florida, Tampa, Fla., USA}

Purpose: A prospective, randomized, clinical study of transjugular intrahepatic portasystemic shunt (TIPS) versus operative portacaval shunt with a small-diameter $8 \mathrm{~mm}$ graft (HGPCS) as definitive treatment of bleeding varices from portal hypertension.

Materials and Methods: 140 patients were enrolled in the study during the period 1993-2003. Both shunts were undertaken as 
definitive therapy and never as a 'bridge to transplantation'. All patients had bleeding esophageal/gastric varices and failed or could not undergo sclerotherapy/banding. Patients with portal vein thrombosis or hopeless survival were excluded from the study. Failure of shunting was defined as inability to shunt, irreversible shunt occlusion, major variceal rehemorrhage, liver transplantation or death. Median postoperative follow-up was 64 months (range 6-96 months). Statistical analysis of the results was achieved with Fischer's exact test.

Results: There were 70 patients in each group, with no difference in age, gender, Child's class, etiology of cirrhosis, urgency of shunting, or incidence of ascites or encephalopathy between groups. TIPS reduced portal pressures from $32 \pm 7.5 \mathrm{mmHg}$ to $25 \pm 7.5 \mathrm{mmHg}(\mathrm{p}<0.01)$, whereas HGPCS reduced them from $30 \pm 4.6 \mathrm{mmHg}$ to $19 \pm 5.3 \mathrm{mmHg}(\mathrm{p}<0.01)$. TIPS could not be placed in five patients. Shunt stenosis/occlusion was more frequent after TIPS. As of that, 45 (64\%) patients failed after TIPS, whereas after H-graft portacaval shunt $18(26 \%)$ patients failed $(\mathrm{p}<0.05)$. Recurrence of variceal bleeding, liver transplantation and death were reported more frequently after TIPS $(\mathrm{p}<0.01)$.

Conclusions: Both TIPS and HGPCS reduced portal pressure. Placement of TIPS, however, resulted in more deaths, greater risk for rebleeding and more frequent treatment failures. Mortality and treatment failure rates promote the application of HGPCS over TIPS.

\section{9}

\section{Comparison of Two New Techniques for Liver Transection during Hepatectomy: Radiofrequency Energy vs Ligasure Sealing System - An Animal Study}

\section{K. Tepetes, M. Spyridakis, T. Theothoropoulos, C. Hatzitheofilou}

Department of Surgery, University Hospital of Larissa, Larissa, Greece

In the reported animal study 6 pigs weighting $20-25 \mathrm{~kg}$ were used. Overall, 12 hepatic lobectomies were carried out.

Four pigs (Group A) underwent two lobectomies each with the transection plane coagulated prior to the division with a modification of the cluster cool-tip Radionics radiofrequency electrode. In the remaining two pigs (Group B) the liver resections were performed using the Atlas Ligasure clamp.

The blood loss per lobectomy in Group A varied 5-10 ml (mean: 7) whereas in Group B the blood loss was 30-50 ml (mean: 42).

Even if the study is not completed yet in order to draw statistically significant conclusions, it seems that the application of the radiofrequency energy in liver resections is associated with less blood loss than the use of the Ligasure vessel sealing device.
20

\section{The Impact of Moderate Hypothermia on Haemostasis: Experimental Study - Preliminary Data}

\author{
Ch. Staikou', M. Kontos, E. Drakos², E. Papaioannou', \\ I. Papakonstantinou, E. Bastounis \\ 1st Department of Surgery, University of Athens, \\ 'Department of Anaesthesia, '2Laboratory of Pathology, \\ 'Laikon General Hospital of Athens', Athens, Greece
}

Purpose: The purpose of this study was to investigate the effect of moderate hypothermia on natural inhibitors of coagulation: antithrombin III (ATIII), and protein C (PC), factors of fibrinolytic system (plasminogen - PLG), and vascular wall (aorta and inferior vena cava).

Materials and Methods: Ten male New Zealand rabbits were subjected to moderate hypothermia of $32^{\circ} \mathrm{C}$. The rabbits were shaved and taken to the operating theatre (environmental temperature of $22-24^{\circ} \mathrm{C}$ ). They were anaesthetized and a tracheotomy was performed for continuous mechanical ventilation under anaesthesia. Cooling was achieved by applying icepacks directly to the skin. The animals were kept at a steady core temperature (oesophageal thermistor) of $32^{\circ} \mathrm{C}$ for $60 \mathrm{~min}$. After that period, blood samples and tissue specimens were obtained. Heparin was strictly excluded from the experimental protocol. ATIII, PC and PLG were determined by chromogenic assay methods and compared to normothermic samples of the same animal obtained a few days before.

Tissue specimens (aortic and inferior vena cava wall) were examined under light microscope. Paired t-test was used for the statistical analysis ( $\mathrm{P}<0.05$ was considered statistically significant).

Results: ATIII and PC were significantly reduced, while PLG was increased.

\begin{tabular}{lcll}
\hline & Normothermia & Hypothermia & $\begin{array}{l}\text { Statistical } \\
\text { significance }\end{array}$ \\
\hline ATIII (\%) & $103 \pm 12$ & $83 \pm 20$ & $\mathrm{P}=0.01$ \\
PC (\%) & $70 \pm 7$ & $53 \pm 7$ & $\mathrm{P}<0.005$ \\
PLG (\%) & $14 \pm 0.5$ & $17 \pm 2$ & $\mathrm{P}=0.006$ \\
\hline
\end{tabular}

No significant lesions were observed on the vessel wall under the light microscope.

Conclusions: This study suggests that even though the hypothermia period was relatively short, haemostatic mechanism was impaired. Our data indicate an increased thrombotic and fibrinolytic tendency, without significant vessel wall lesions. Major trauma and abdominal surgery are related to hypothermia, usually of a duration longer than $60 \mathrm{~min}$. Coagulation defects are relatively early effects of hypothermia; they should be prevented and corrected promptly, well before standard laboratory tests or clinical signs indicate the disorder. 


\section{1 \\ Molecular Markers (PECAM-1, ICAM-3, HLA-DR) Determine Prognosis in Primary Non-Hodgkin's Gastric Lymphoma Patients}

A. Darom'1, I.P. Gomatos', E. Leandros', E. Chatziyianni', M.M. Konstadoulakis', C. Dervenis², G. Androulakis ${ }^{1}$

${ }^{1}$ Laboratory of Surgical Research, First Department of Propaedeutic Surgery, Hippokration Hospital, Athens Medical School, ${ }^{2}$ A Department of Surgery, St Olga Hospital, Athens, Greece

Background: Primary gastric non-Hodgkin's lymphomas represent uncommon tumors of the gastrointestinal tract associated with a poor clinical outcome.

Aim: To evaluate the prognostic significance of PECAM-1, ICAM-3 and HLA-DR antigen expression in patients with primary non-Hodgkin gastric lymphoma.

Patients and Methods: We immunohistochemically studied PECAM-1, ICAM-3 and HLA-DR antigen expression in 36 gastric lymphoma patients. They were all diagnosed and treated in our Department from 1990 to 1995 . Ten non-malignant gastric tissue specimens and ten healthy gastric tissue specimens were used as controls. Clinicopathological and survival data were correlated with the staining results.
Results: HLA-DR antigen expression was detected in 33 gastric lymphoma patients $(91.7 \%)$ and 6 non-malignant cases $(54.54 \%)$. PECAM-1 stained the tumor cells of 10 patients $(27.8 \%)$, the endothelial cells of 9 patients $(25 \%)$ and the inflammatory infiltrate of 4 patients (40\%) with benign gastric disease. ICAM-3 expression was observed on the tumor cells of 17 patients (47.2\%), while 5 nonmalignant cases $(50 \%)$ stained positive as well. None of the healthy controls stained for any of the genes studied.

In the multivariate analysis, HLA-DR antigen and PECAM-1 proved to be statistically significant independent prognostic factors associated with a favorable and an unfavorable prognosis respectively $(\mathrm{p}=0.0029$ and $\mathrm{p}=0.0088)$. In the univariate analysis, patients who were PECAM-1(+)/ICAM-3(-) or HLA-DR $(-) / \operatorname{ICAM}-3(-)$ exhibited a significantly decreased overall survival compared to those with the exactly opposite expression patterns $(\mathrm{p}=0.0041$ and $\mathrm{p}=0.0091$, respectively). Those patients who were HLA-DR(+)/ ICAM$3(+) /$ PECAM-1 $(-)(\mathrm{n}=8)$ had a 100\% 5-year survival rate which was significantly higher compared to the rest of the group $(n=24)$ $(\mathrm{p}=0.0289)$.

Conclusions: PECAM-1, ICAM-3 and HLA-DR are representative markers of tumor expansion potential and host immune surveillance respectively. Their study on tissue specimens depicts not only the specific tumor aggressiveness, but also the intensity of antitumor response. Their combined use may help us to identify high-risk patients who could benefit from more aggressive therapeutic protocols. 


\section{Digestive Surgegeriy}

Anagnostoulis, S. 7

Andreou, J. 13

Androulakis, G. 3, 21

Assimakopoulos, S.F. 9

Bassi, C. 8

Bastounis, E. 1, 2, 20

Baziotis, N. 6

Beger, H.G. 8

Bolanaki, H. 7

Bontozoglou, N. 12

Bramis, J. 1, 2

Braumann, C. 14

Büchler, M.W. 8

Carey, L.C. 17, 18

Charonis, A. 9

Chatziyianni, E. 21

Chrysikopoulos, H. 13

Cuzzocrea, S. 11

Darom, A. 21

Dervenis, C. 8, 21

Doumaki, H. 5

Drakos, E. 20
Efthimiadou, A. 7

Efthimiadou, R. 13

Eleftheriadis, E. 4, 5

Friess, H. 8

Georgiou, C. 9

Goldin, S.B. 16

Gomatos, I.P. 21

Gorgoulis, V.G. 1

Hatziyianni, E. 3

Hatzitheofilou, C. 19

Hatzitheoklitos, E. 8

Heliadis, S. 4

Hickey, H. 8

Jacobi, C.A. 14

Kalathaki, O. 3

Kapoutzis, K. 5

Karagiannis, D. 12

Karayiannakis, A. 7

Kolios, G. 4

Konstadoulakis, M.M. 21

Konstantinidis, K. 12

Kontos, M. 20

Korkolis, D.P. $15-18$
Kotsinas, A. 1

Kotzampassi, K. 4, 5

Leandros, E. 21

Leonardou, P. 1, 2

Maniatis, V. 13

Markoyiannakis, H. 3

Mathou, N. 12

Memos, N. 3

Menenakos, C. 14

Metaxas, G. 4, 5

Mueller, J.M. 14

Murr, M.M. 15

Mylonaki, D.B. 3

Neoptolemos, J.P. 8

Nikolaou, A. 2

Nikolopoulou, V. 9

Papaioannou, E. 20

Papakonstantinou, I. 1, 2, 20

Pappas, J. 13

Paramythiotis, D. 4, 5

Paraskeuopoulos, K. 12

Polychronidis, A. 7

Ramandanis, G. 6

Rosemurgy, A.S. 15-18
Roussakis, A. 13

Rueckert, J.I. 14

Savidou, D. 13

Scopa, C.D. 9

Sigala, F. 1

Simopoulos, C. 7

Siriwardena, A.K. 10, 11

Sotiropoulos, V. 12

Spyridakis, M. 19

Staikou, Ch. 20

Stocken, D.D. 8

Striggaris, K. 2

Tepetes, K. 19

Theothoropoulos, T. 19

Tsamakidis, K. 6

Tsavaris, N. 2

Vagianos, C. 9

Vardaki, E. 13

Virlos, I.T. 10, 11

Zacharatos, P. 1

Zervos, E.E. 15-18

Zissimopoulos, A. 6 